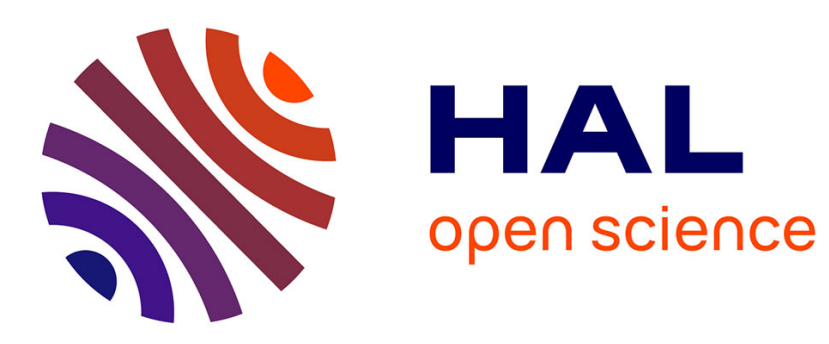

\title{
Resolving Triblock Terpolymer Morphologies by Vapor-Phase Infiltration
}

\author{
Sangho Lee, Ashwanth Subramanian, Nikhil Tiwale, Kim Kisslinger, \\ Muhammad Mumtaz, Ling-Ying Shi, Karim Aissou, Chang-Yong Nam, \\ Caroline A Ross
}

\section{To cite this version:}

Sangho Lee, Ashwanth Subramanian, Nikhil Tiwale, Kim Kisslinger, Muhammad Mumtaz, et al.. Resolving Triblock Terpolymer Morphologies by Vapor-Phase Infiltration. Chemistry of Materials, 2020, 32 (12), pp.5309-5316. 10.1021/acs.chemmater.0c01647. hal-03040132

\section{HAL Id: hal-03040132 https://hal.science/hal-03040132}

Submitted on 4 Dec 2020

HAL is a multi-disciplinary open access archive for the deposit and dissemination of scientific research documents, whether they are published or not. The documents may come from teaching and research institutions in France or abroad, or from public or private research centers.
L'archive ouverte pluridisciplinaire HAL, est destinée au dépôt et à la diffusion de documents scientifiques de niveau recherche, publiés ou non, émanant des établissements d'enseignement et de recherche français ou étrangers, des laboratoires publics ou privés. 


\section{Resolving Triblock Terpolymer Morphologies by Vapor-Phase Infiltration}

Sangho Lee ${ }^{1}$, Ashwanth Subramanian ${ }^{2}$, Nikhil Tiwale ${ }^{3}$, Kim Kisslinger ${ }^{3}$, Muhammad Mumtaz $^{4}$, Ling-Ying Shi ${ }^{1,5}$, Karim Aissou ${ }^{4,6}$, Chang-Yong Nam ${ }^{2,3}$, and Caroline A. Ross ${ }^{1 *}$

${ }^{1}$ Department of Materials Science and Engineering, Massachusetts Institute of Technology, Cambridge MA 02139, USA

${ }^{2}$ Department of Materials Science and Chemical Engineering, Stony Brook University, Stony Brook, New York 11794, USA

${ }^{3}$ Center for Functional Nanomaterials, Brookhaven National Laboratory, New York 11973, USA

${ }^{4}$ Laboratoire de Chimie des Polymères Organiques (LCPO), CNRS - ENSCPB - Université de Bordeaux, Pessac Cedex, France

${ }^{5}$ College of Polymer Science and Engineering, State Key Laboratory of Polymer Materials Engineering, Sichuan University, Chengdu 610065, China

${ }^{6}$ Institut Européen des Membranes, Université de Montpellier-CNRS—ENSCM, 300 Avenue du Professeur Emile Jeanbrau, F-34090 Montpellier, France

*E-mail: caross@mit.edu 
ABSTRACT: The spontaneous formation of well-organized three-dimensional (3D) nanostructures from self-assembled block copolymers (BCPs) holds promise for nanofabrication and lithography. The addition of a third block to BCPs provides access to a plethora of 3D geometries, but it remains difficult to resolve the geometry of such "three-color" structures when there is low contrast between the polymeric components at length scales of a few nm. Here, we apply vapor-phase infiltration synthesis to a silicon-containing triblock terpolymer, poly(1,1dimethyl silacyclobutane- $b$-styrene- $b$-lactide) (PDMSB- $b$-PS- $b$-PLA or DSL) to distinguish the 3D microdomain morphologies of the two organic blocks, PLA and PS. Selective infiltration of $\mathrm{ZnO}$ within the PLA microdomains reveals morphologies consisting of three-color lamellae or lamellae combined with vertically aligned core-shell cylinders, depending on the volume fractions of each block. The infiltration produces $\mathrm{ZnO}$ nanoparticles throughout the $260 \mathrm{~nm}$ thickness of the DSL film, generating 3D nanocomposites containing $\mathrm{ZnO}$ and $\mathrm{SiO}_{\mathrm{x}}$. These results provide a strategy for synthesizing multicomponent 3D nanostructures as well as visualizing the phase behavior of multi-block copolymers.

KEYWORDS: three-dimensional self-assembly, triblock terpolymer, infiltration synthesis, metal oxide, three-color nanostructure. 


\section{INTRODUCTION}

Three-dimensional (3D) nanofabrication is essential for producing a host of useful materials and structures for applications such as electronic devices, nanoporous membranes and functionalized surfaces ${ }^{1-6}$ Self-assembly of block copolymer (BCP) thin films in particular offers an efficient route to produce a variety of 3D nanostructures, taking advantage of its simple sequence of processing steps. ${ }^{7-14}$ Compared with diblock copolymers, triblock and multiblock polymers can produce nanostructures with greater structural diversity and additional chemical functionalities, ${ }^{15-20}$ and there has been an increasing emphasis on understanding their selfassembly and 3D morphologies..$^{21-25}$

However, it is challenging to resolve the morphologies of 3D BCPs comprising more than two polymeric components unless there is good contrast between the microdomains, for example a contrast in density, chemical composition, or reactivity. Block-selective etching followed by planview or cross-sectional imaging using scanning electron microscopy (SEM) is commonly used to characterize $\mathrm{BCP}$ morphologies, but the organic blocks are often difficult to distinguish. ${ }^{23,26} \mathrm{X}$-ray scattering is used for 3D structural analysis, but it requires a scattering contrast between the blocks, for example the presence of metal or $\mathrm{Si}$ in one block, ${ }^{13,23}$ and analysis of triblock morphologies (beyond measuring the periodicity) requires modeling the structure factor.

Vapor-phase infiltration (VPI) refers to variations of ex situ material hybridization techniques based on atomic layer deposition (ALD) including multi-pulse infiltration, ${ }^{27}$ sequential vapor infiltration (SVI), ${ }^{28}$ and sequential infiltration synthesis (SIS), ${ }^{29}$ where vapor-phase inorganic precursors perfuse the polymer matrix to generate unique organic-inorganic hybrids with improved material properties. When applied to a self-assembled $\mathrm{BCP}$, it can greatly enhance the scattering contrast and the etch resistance between blocks by selectively incorporating inorganic material 
such as $\mathrm{ZnO}, \mathrm{TiO}_{2}$, or $\mathrm{Al}_{2} \mathrm{O}_{3}$ into specific microdomains. ${ }^{27-37}$ The infiltration process proceeds via sorption (diffusion) and block-selective reaction of vapor-phase organometallic precursors into the polymer blocks with suitable reactive moieties to generate infused inorganic materials within the target BCP microdomains. ${ }^{38-39}$ The hybrid nanocomposite thus formed can also be converted to an inorganic nanostructure inheriting the original structure of the polymer microdomains, ${ }^{34,36}$ not only producing various functional nanostructures in a simple process but also enabling all-organic $\mathrm{BCP}$ morphologies to be fully resolved. ${ }^{31}$ ALD of metal oxide precursors into cylinder- and gyroidforming BCPs has been used to generate 1D and 3D nanostructures for a broad variety of nanotechnologies such as gas sensors, solar cells, photocatalysts, and pattern transfer masks. ${ }^{14,32,}$ $35,40-42$

While the majority of the reports exploiting infiltration synthesis of BCPs are focused on diblock copolymers, the process is also applicable to BCPs containing three or more blocks such as triblock terpolymers (TBTPs). ${ }^{37}$ In this work we demonstrate the infiltration of inorganic species into poly(1,1-dimethyl silacyclobutane-block-styrene-block-lactide) (PDMSB- $b$-PS- $b$-PLA or DSL), a silicon-containing TBTP with two organic blocks, PS and PLA. Our previous study of one composition of DSL reported the formation of microdomains consisting of core-shell cylinders perpendicular to perforated lamellae, based on SEM and grazing-incidence small-angle X-ray scattering (GISAXS) characterization. ${ }^{23}$ The PDMSB was clearly resolved but it was difficult to differentiate between the PLA and the PS blocks. In this work, we demonstrate the effects of $\mathrm{ZnO}$ infiltration into a series of DSL compositions that includes both lamellar and lamellar plus hexagonal cylinder morphologies. We first investigate the phase behavior of four DSLs with different volume fractions of each block and then show that infiltration synthesis introduces $\mathrm{ZnO}$ 
into the PLA microdomains. These results not only reveal the microdomain morphologies of the DSL, but demonstrate the formation of nanocomposites with $\mathrm{ZnO}$, silica and organic components. 


\section{RESULTS AND DISCUSSION}
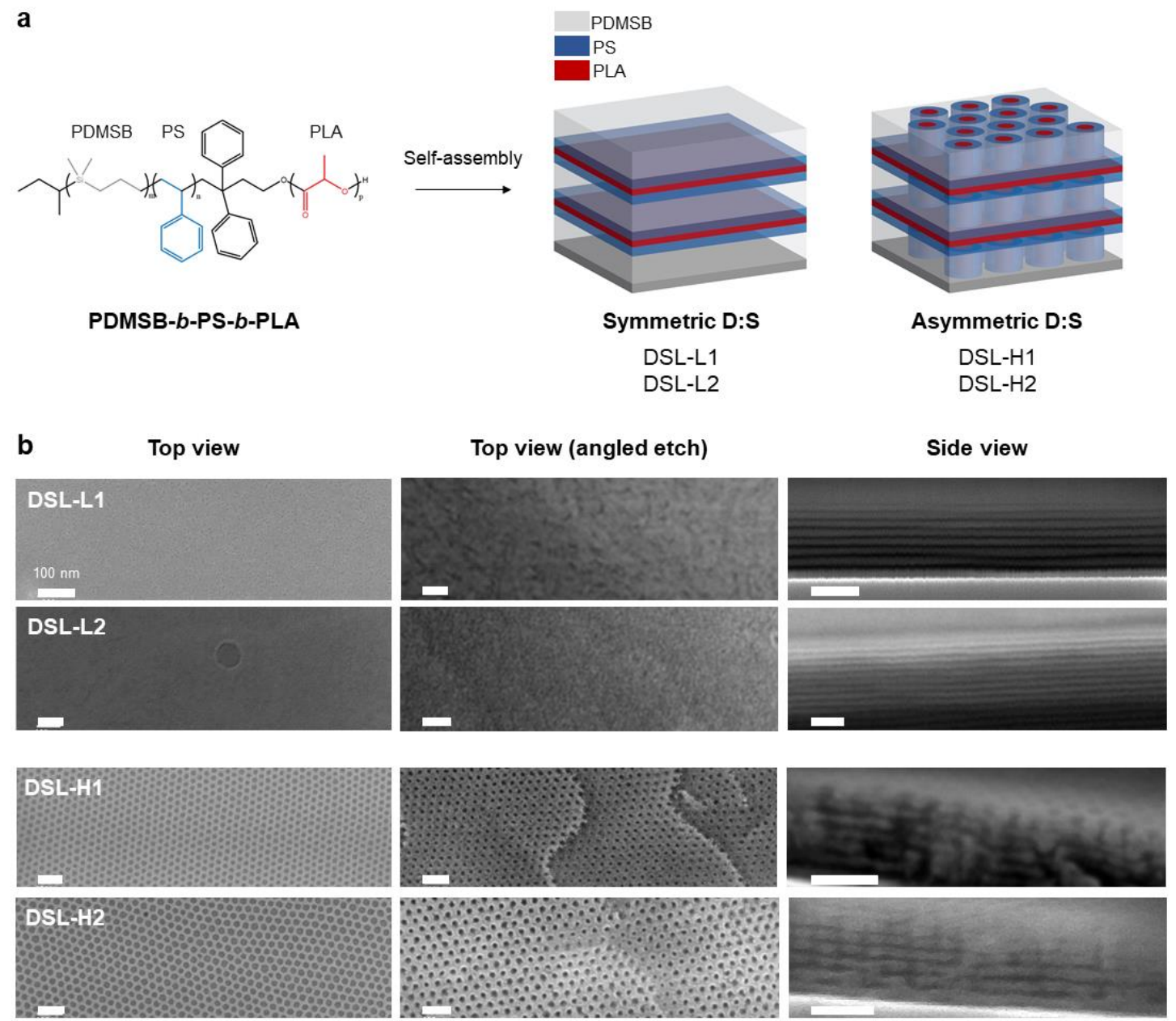

Figure 1. (a) Chemical structure of DSL and schematic illustrations of self-assembled DSL films.

The dominant morphologies obtained in this study for each DSL are presented. DSL-L with symmetric PDMSB and PS compositions form alternating in-plane lamellae (left) and DSL-H with asymmetric PDMSB and PS fractions exhibit in-plane lamellae combined with hexagonally packed vertical core-shell cylinders (right). Schematics in light grey, blue, and red represent PDMSB, PS, and PLA, respectively. (b) SEM images of the final morphologies in DSL films: Top view (left), plan view of terraced layers (middle), and side view (right). The images mainly show contrast from the oxidized PDMSB nanopatterns. 
DSL (Figure 1a) was synthesized using anionic polymerization combined with ring opening polymerization as described in the Methods section and Ref (23). We previously described the syntheses of PDMSB- $b$-PS- $b$-poly(methyl methacrylate) (DSM) ${ }^{23,37,43}$ and PDMSB- $b$-PS- $b$-poly(2-vinylpyridine) (DSV). ${ }^{25}$ The PDMSB (not to be confused with PDMS, polydimethylsiloxane) monomeric unit is composed of butane with one Si atom in the backbone. DSL films of 180-260 $\mathrm{nm}$ thicknesses were spin cast onto Si substrates and annealed in a solvent vapor flow of $\mathrm{CHCl}_{3}$. The organic blocks were etched by oxygen plasma reactive ion etching (RIE) and both top and side views of the structure were characterized by SEM. Further analysis was carried out using transmission and scanning transmission electron microscopy (TEM/STEM) and elemental mapping using energy dispersive spectroscopy (EDS). The bulk morphology of vacuum annealed samples was characterized using small angle x-ray scattering (SAXS).

Table 1. DSL properties, morphologies, and periods in thin films.

\begin{tabular}{|c|c|c|c|c|c|c|}
\hline & $\begin{array}{c}\text { Molecular } \\
\text { weight, } \mathrm{M}_{\mathrm{n}} \\
{[\mathrm{kg} / \mathrm{mol}]}\end{array}$ & $\begin{array}{l}\text { Dispersity, } \\
\mathrm{M}_{\mathrm{w}} / \mathrm{M}_{\mathrm{n}}\end{array}$ & $\begin{array}{l}\text { Composition, } \\
f_{\mathrm{PDMSB}} / f_{\mathrm{PS}} / f_{\mathrm{PLA}}\end{array}$ & $\begin{array}{c}\text { Thin film } \\
\text { thickness } \\
\text { [nm] }\end{array}$ & $\begin{array}{l}\text { Thin film } \\
\text { morphology }\end{array}$ & $\begin{array}{l}\text { Period } \\
{[\mathrm{nm}]^{\mathrm{a})}}\end{array}$ \\
\hline DSL-L1 & 28.4 & 1.15 & $0.41 / 0.40 / 0.19$ & 180 & $\mathrm{Lam}_{/ /}$ & 21 \\
\hline DSL-L2 & 25.8 & 1.12 & $0.36 / 0.36 / 0.28$ & 210 & $\mathrm{Lam}_{/ /}$ & 16 \\
\hline DSL-H1 & 55.5 & 1.09 & $0.25 / 0.40 / 0.35$ & 240 & $\operatorname{Lam}_{/ /-\mathrm{Hex}_{\perp}}$ & 21,38 \\
\hline DSL-H2 & 38.4 & 1.07 & $0.31 / 0.53 / 0.16$ & 260 & $\operatorname{Lam}_{/ /-\mathrm{Hex}_{\perp}}$ & 17,35 \\
\hline
\end{tabular}


Four DSL compositions were used in this study (Table 1). DSL-L1 and DSL-L2 had symmetric PDMSB and PS volume fractions, while DSL-H1 and DSL-H2 had a volume fraction of PS that was $~ 1.5$ times that of PDMSB. DSL-H2 is the same polymer composition reported in Ref (23). The SAXS data for the bulk DSLs are shown in Figure S1. The SAXS contrast originates from the difference in scattering between the PDMSB and the organic blocks. DSL-L1 showed lamellar (Lam) and DSL-L2 showed hexagonally packed cylindrical (Hex) morphologies with period $L_{0}=$ $23.2 \mathrm{~nm}$ and $31.4 \mathrm{~nm}$ respectively. DSL-H1 exhibited broad peaks, due to its larger molecular weight, and has been tentatively indexed to hexagonally-perforated lamellae (HPL) with an AB stacking $\left(L_{100}=39.2 \mathrm{~nm}\right)$, i.e. HPL with the perforations in one layer located above the junctions in the adjacent layers, while DSL-H2 showed peaks from a mixture of Hex $\left(L_{0}=19.0 \mathrm{~nm}\right)$ and $\operatorname{Lam}\left(L_{0}=36.9 \mathrm{~nm}\right)$ structures.

SEM images of DSL films after oxygen RIE are shown in Figure 1b. In both DSL-L1 and L2, the plan view images show no periodic features but in-plane lamellae $(\mathrm{Lam} /)$ are observed in the cross-sectional images. This technique does not reveal any contrast between PS and PLA microdomains, showing only the PDMSB lamellae alternating with the non-Si-containing blocks. The Lam structure agrees with the bulk morphology of the DSL-L1, but the DSL-L2 was Hex in bulk, and the Lam thin film structure may be a result of the preferential swelling of PDMSB domains by $\mathrm{CHCl}_{3}$ during the solvent annealing leading to an effective increase in volume fraction. Note that differences between the bulk and thin film morphologies have also been observed for both $\mathrm{DSV}^{25}$ and $\mathrm{DSM}^{43}$ samples.

For the DSL-H1 and H2 films, the top view SEM images (Figure 1b and S2) clearly exhibit a hexagonal pore array attributed to out-of-plane cylindrical morphology, Hex $\_$. The pores show a core-shell morphology assumed to consist of a PS shell and a PLA core, evident from a difference 
in their etching rate in the $\mathrm{O}_{2}$ plasma. To examine the structure at different depths, we introduced an angled plasma RIE followed by SEM imaging from the top surface (see Methods). This reveals terraces showing vertical alignment of the core-shell cylinders throughout the film, in agreement with the DSL-H2 results in Ref (23). Cross-section imaging of both DSL-H1 and H2 show inplane Lam// with vertical features corresponding to the cylinders passing through the perforations in the lamellae. We therefore describe the structure as Lam//-Hex 1 . Our earlier work ${ }^{23}$ on DSL-H2 also showed about $20 \%$ of the film had a morphology attributed to alternating in-plane cylindrical microdomains (Alt//); a similar result was found in the present work for DSL-H2 but no in-plane cylinders were found in DSL-H1.

To clarify the distribution of PS and PLA within the microphase-separated DSL films, we employed infiltration synthesis to infuse $\mathrm{ZnO}$ selectively within the PLA microdomains. $\mathrm{ZnO}$ infiltration consisted of cyclic exposures of the BCP film to diethylzinc (DEZ) and water, which are precursors respectively for $\mathrm{Zn}$ and $\mathrm{O}$. In total 6 cycles were performed, assisted by an initial single $\mathrm{AlO}_{\mathrm{x}}$ infiltration cycle using trimethylaluminum (TMA) and water (referred to as $\mathrm{AlO}_{\mathrm{x}}$ priming). ${ }^{30,44-45}$ During an infiltration synthesis cycle, gaseous organometallic precursors, which are typically Lewis acids, diffuse into the polymer matrix and react with Lewis-basic functional groups within the polymer to form chemical bonds. A subsequent exposure of the film to an oxidizing agent, such as water, transforms the pre-infiltrated organometallic precursor into metal oxide molecules. In the DSL films, only the PLA block contains a Lewis-basic carbonyl group and, thus, metal oxide moieties are expected to be selectively infiltrated into the PLA domain. However, similar to the well-studied PMMA, the carbonyl group within PLA does not readily react with weakly Lewis-acidic DEZ. ${ }^{30,34,44-45}$ This was mitigated by the $\mathrm{AlO}_{\mathrm{x}}$ priming, because the more Lewis-acidic TMA can react better with the carbonyl group and generate -Al-OH species in the 
PLA domain once the priming cycle is completed with water. The available $\mathrm{OH}$ group then can readily react with DEZ, facilitating the subsequent infiltration cycles of $\mathrm{ZnO}$.

a

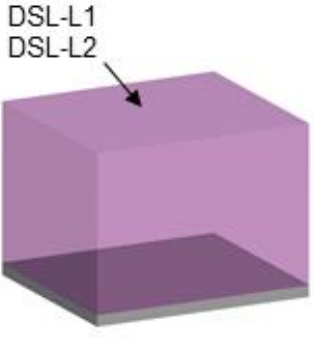

Spin-coated DSL

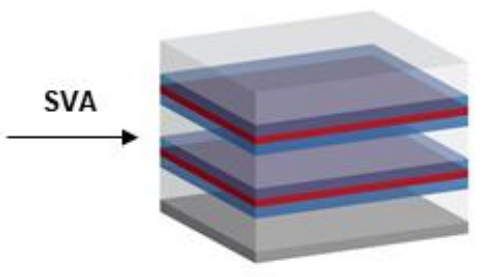

Self-assembled DSL

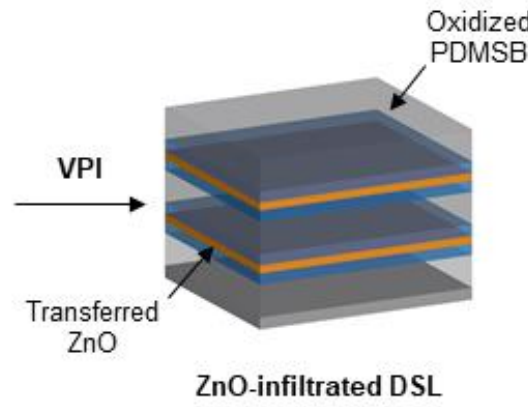

ZnO-infiltrated DSL

b
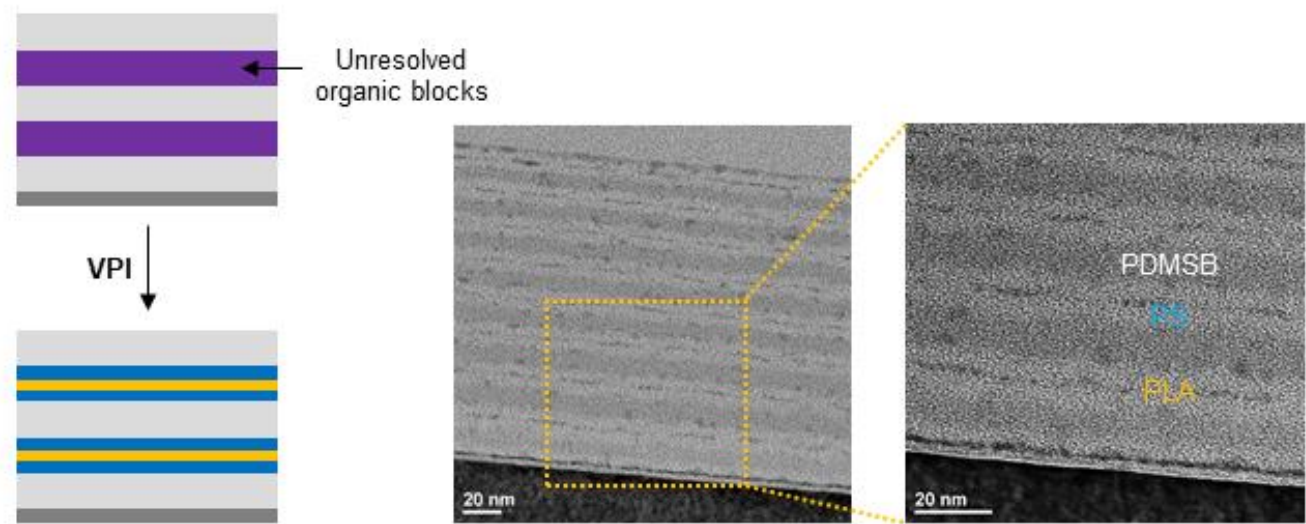

C
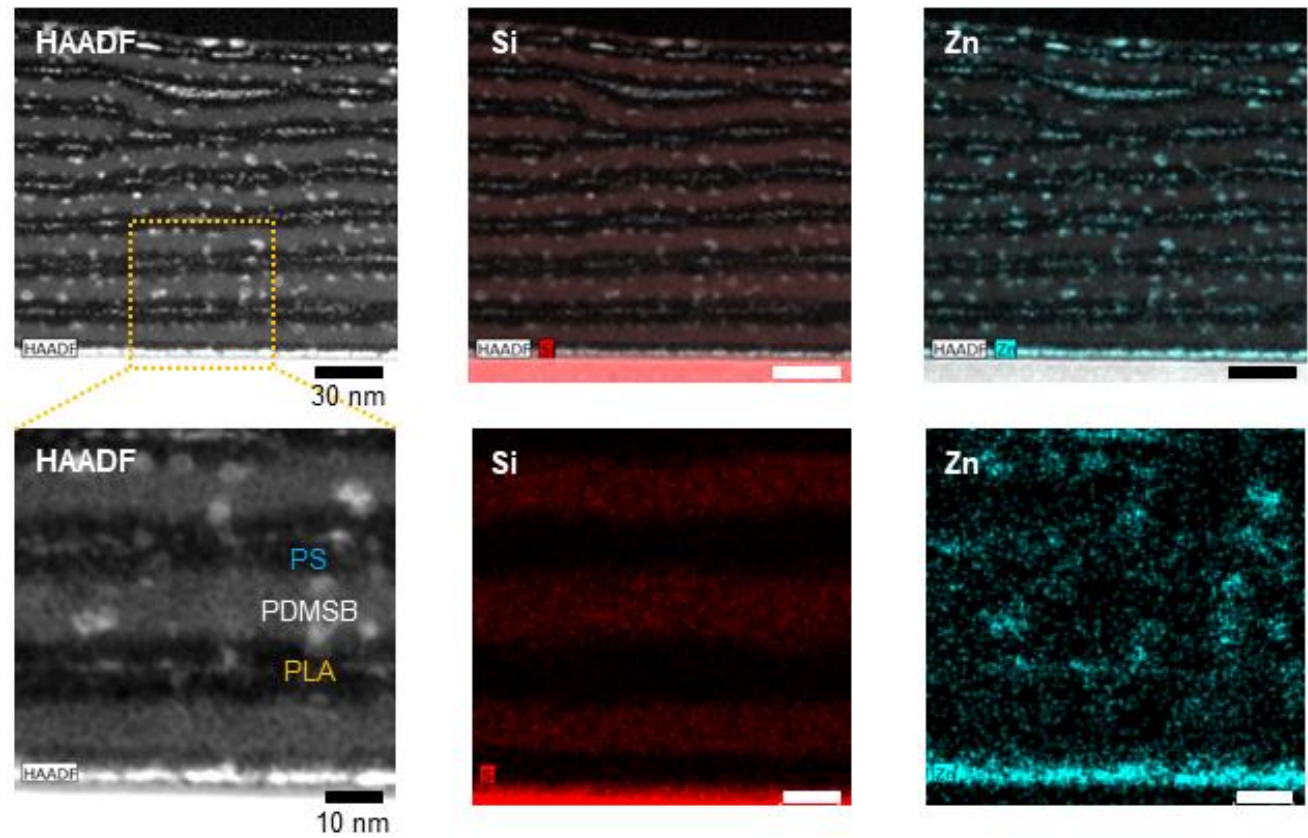
Figure 2. (a) Schematic illustrations depicting the solvent vapor annealing (SVA) followed by infiltration synthesis to produce $\mathrm{SiO}_{\mathrm{x}} / \mathrm{PS} / \mathrm{ZnO}$ nanostructures in DSL-L films. The purple structure in the spin-coated DSL film represents as-cast DSL-L before microphase separation. Schematics in light grey, blue, red, grey, and yellow represent PDMSB, PS, PLA, $\mathrm{SiO}_{\mathrm{x}}$, and $\mathrm{ZnO}$, respectively. (b) Schematic illustrations (left) of the cross-sections of DSL-L structure before (top) and after (bottom) infiltration of $\mathrm{ZnO}$ and TEM images (right) of resultant $\mathrm{SiO}_{\mathrm{x}} / \mathrm{PS} / \mathrm{ZnO}$ nanostructure in DSL-L1 film. (c) HAADF STEM images and associated EDS elemental mappings ( $\mathrm{Si}$ and $\mathrm{Zn}$ ) for cross-sections of ZnO-infiltrated DSL-L1.

Figure 2a shows schematics of the proposed structure of the DSL-L thin films after infiltration, with $\mathrm{ZnO}$ present in the PLA blocks. Figure $\mathbf{2 b}$ shows a cross-sectional bright-field TEM image of DSL-L1 after ZnO infiltration. The dark and uniform in-plane lamellae consist of the Sicontaining PDMSB block. However, additional layers are evident within the in-plane lamellae which are identified as $\mathrm{ZnO}$-infused PLA, confirming the alternating 3-color lamellar structure of DSL-L1. DSL-L2 shows similar morphology, shown in Figure S3 and S4. The ZnO-infiltrated PLA lamellae exhibit rough interfaces and a sparse distribution of $\mathrm{ZnO}$ particles, which is attributed to the small volume fraction of PLA $\left(f_{\mathrm{PLA}}=0.19\right)$ in DSL-L1. The discontinuous $\mathrm{ZnO}$ growth inside the PLA domain could also result from the single $\mathrm{AlO}_{\mathrm{x}}$-priming cycle that is insufficient to provide uniform reactivity with the infiltrating DEZ; we did not pursue $\mathrm{AlO}_{\mathrm{x}}$-only infiltration due to the lack of $\mathrm{Z}$-contrast between $\mathrm{Al}$ and $\mathrm{Si}$, but this was demonstrated previously for DSM where the PMMA cores of core-shell cylinders were decorated with alumina. ${ }^{37}$ DSL-L2 which includes a larger fraction of PLA $\left(f_{\mathrm{PLA}}=0.28\right)$ exhibits more homogeneous $\mathrm{ZnO}$ layers in between PS lamellae (Figure S3 and S4). 
The ZnO-infiltrated DSL-L1 structure was analyzed by high-angle annular dark-field (HAADF) imaging and EDS elemental mapping (Figure 2c). HAADF images sensitive to Z-contrast reveal the three-color lamellar structure with $\mathrm{ZnO}$ present in the PLA throughout the film thickness. This demonstrates the potential of this approach to resolve the internal structure of even thick films. Elemental mapping shows that as expected, Si is uniformly distributed in the PDMSB domain which exhibit sharp interfaces, but oxygen is also present. In fact, the oxidation appears to have occurred before the $\mathrm{ZnO}$ infiltration (Figure S5), likely due to ambient oxygen and water. The majority of $\mathrm{Zn}$ is selectively infiltrated into PLA but there are also $\mathrm{ZnO}$ particles present in the PDMSB, presumably due to its prior oxidation which could provide an increased reactivity of the PDMSB toward DEZ. Kinetic entrapment of precursors driven by weak physisorption within the polymer matrix might also contribute, as has been proposed to explain $\mathrm{AlO}_{\mathrm{x}}$ and $\mathrm{ZnO}$ infiltration into PDMS. ${ }^{46-48}$ It is noted that the nominally unreactive blocks around the PLA are not expected to retard the infiltration of $\mathrm{ZnO}$ into the targeted PLA domain because the absence of strong interaction between infiltrating precursors and unreactive polymer domains renders their diffusion through the inert domains fast, as confirmed by a recent study by Waldman et al. examining the infiltration of weakly interacting organometallic precursors in homopolymer thin films. ${ }^{49}$ This is consistent with previous reports that have shown a high-fidelity block-selective infiltration of various inorganic materials into isolated PMMA domains surrounded by a majority of unreactive PS domains..$^{30-31}$ 


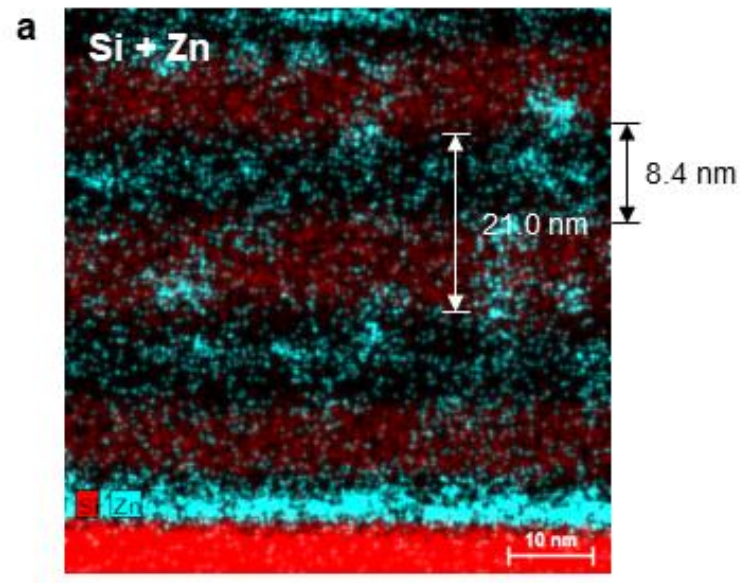

DSL-L1

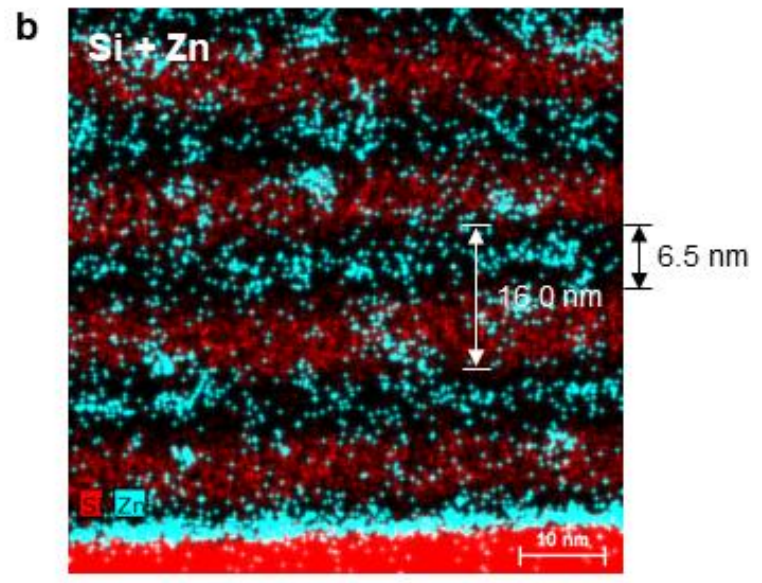

DSL-L2

Figure 3. EDS elemental mappings of both Si (red) and Zn (blue) for DSL structures: (a) DSL-L1 and (b) DSL-L2. Estimated width of each microdomain is indicated.

Based on the elemental maps for the $\mathrm{ZnO}$-infiltrated DSL-L films, we estimated the periodicities and volume fraction of microdomains in each structure as shown in Figure 3. The period of DSL-L1 $\left(L_{0, \mathrm{~L} 1}=21 \mathrm{~nm}\right)$ is larger than that of DSL-L2 $\left(L_{0, \mathrm{~L} 2}=16 \mathrm{~nm}\right), L_{0, \mathrm{~L} 1} / L_{0, \mathrm{~L} 2}=1.31$, though the difference exceeds that predicted from the ratio of (molecular weight $\left.\left(M_{\mathrm{n}}\right)\right)^{2 / 3}$, $\left(M_{\mathrm{n}, \mathrm{L} 1} / M_{\mathrm{n}, \mathrm{L} 2}\right)^{2 / 3}=1.07$. For both structures, the PDMSB domains are 1.5 times wider than the organic domains although the volume fraction of PDMSB is 36-41\%. This is attributed to preferential swelling of the PDMSB during solvent annealing which is at least partly preserved after drying. 

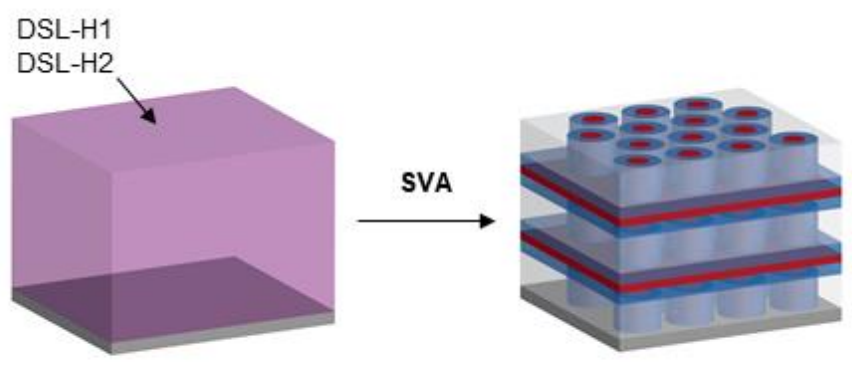

Spin-coated DSL
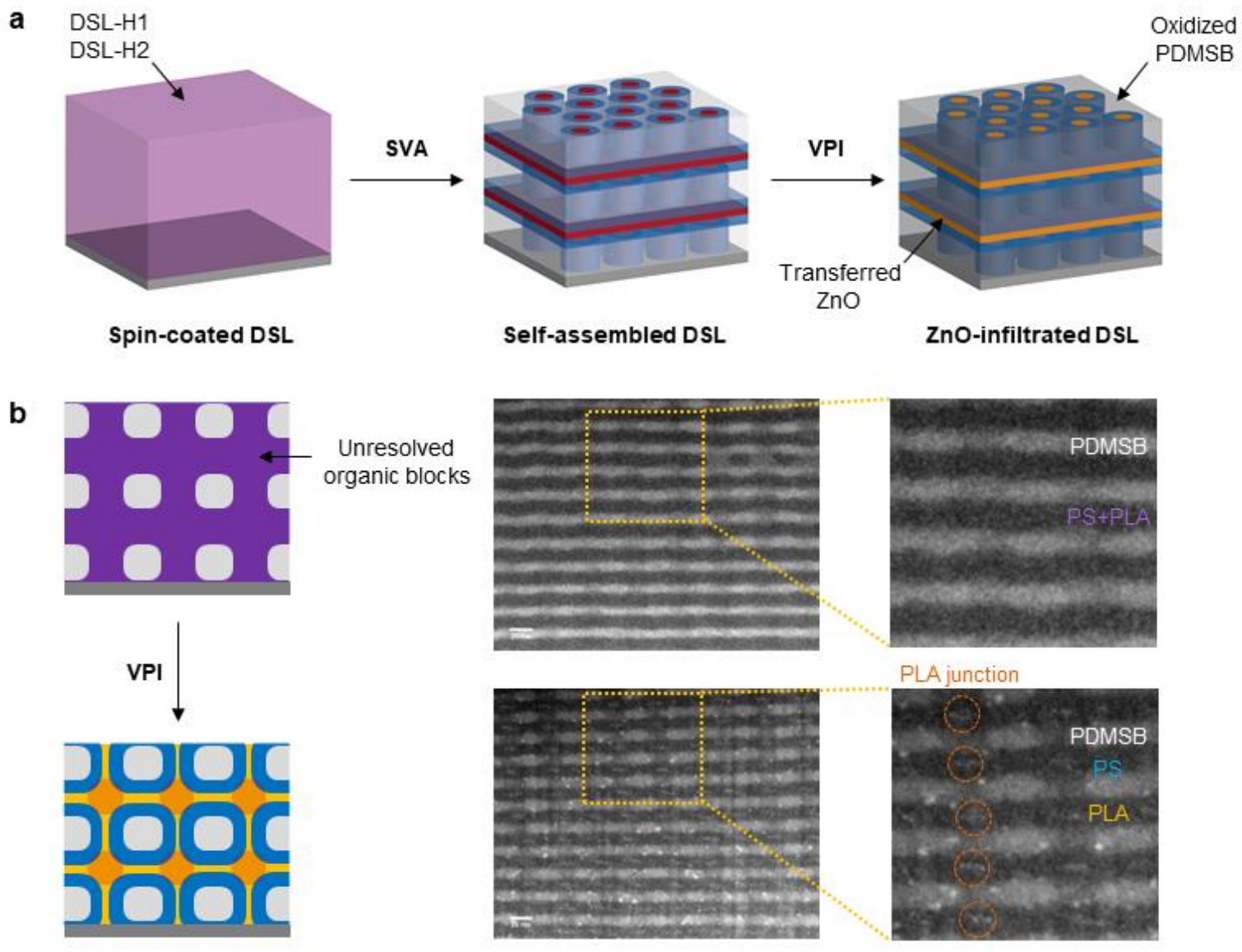

Figure 4. (a) Schematic illustrations depicting SVA followed by infiltration synthesis to produce $\mathrm{SiO}_{\mathrm{x}} / \mathrm{PS} / \mathrm{ZnO}$ nanostructures in DSL-H films. The purple structure in the spin-coated DSL film represents as-cast DSL-H before phase separation. Schematics in light grey, blue, red, grey, and yellow represent PDMSB, PS, PLA, $\mathrm{SiO}_{\mathrm{x}}$, and $\mathrm{ZnO}$, respectively. (b) Schematic illustrations (left) and HAADF STEM images (right) of the cross-sections of DSL-H1 structure before (top) and after (bottom) infiltration of $\mathrm{ZnO}$. The junctions between the lateral and vertical PLA domains are highlighted by orange circles in the schematic and in the magnified STEM image of ZnO infiltrated DSL-H1 structure. 
$\mathrm{ZnO}$ was infiltrated into DSL-H films via the same process as described in Figure 4a. Unlike DSL-L, the DSL-H forms Lam// through which Hex $\perp$ of PLA-PS pass perpendicular to the substrate, i.e. the SEM indicates a morphology consisting of both in-plane and out-of-plane periodic features, a result supported by GISAXS ${ }^{23}$ and also described for thick films of DSV. ${ }^{25}$ Figure 4b shows cross-sectional HAADF STEM data comparing the DSL-H1 morphology before and after $\mathrm{ZnO}$ infiltration. This clearly shows selective $\mathrm{ZnO}$ infiltration into the PLA block at the junction between the lamellae and cylinders, while the un-infiltrated sample exhibits no such contrast. As illustrated in the schematics in Figure $\mathbf{4 b}$, the wider region of $\mathrm{ZnO}$ visible at the cylinder-lamella intersection is attributed to widening of the PLA block at the junction between the cores of the cylinders and the thin PLA lamellae. Little $\mathrm{ZnO}$ is seen outside the junction areas, due to the narrowness of the PLA cores and lamellae. However, it is clear that the $\mathrm{ZnO}$-decorated regions are located at the center of the vertical core-shell cylindrical domains, and the SEM depth profile and cross-section of the etched films (Figures 1b and S2) confirms the continuity of the vertical PLA cores.

The phase behavior of ZnO-infiltrated DSL-H2, which includes much lower fraction of PLA than DSL-H1, is shown in Figure S6. Thin ZnO-decorated layers are present within the PS lamellae corresponding to the PLA lamellae embedded in the PS, as seen in DSL-L1, L2 and H1. This is in agreement with the morphology of DSL-H2 resolved by employing hydrolysis to selectively degrade the PLA domains. ${ }^{23}$ However, unlike DSL-H1, thicker ZnO-infused regions corresponding to the cores of the core-shell cylinders are not evident in the image, even though the SEM plain view and cross-section (Figure 1b) as well as the bright-field cross-section of the un-infiltrated DSL-H2 (Figure S5b) demonstrate continuous vertical cylinders. We assume that the low volume fraction of the PLA limited infiltration of $\mathrm{Zn}$ into the cylinder cores which were 
not resolved in Figure S6. We note that self-assembled BCP films in general can be swelled when exposed to infiltrating precursors as organic vapors can do, ${ }^{50}$ but our TEM data clearly indicates no disruption of the self-assembled BCP morphology by the precursor infiltration. This is most likely due to the purging step included in the infiltration protocol, which removes unreacted precursor out of the BCP film.

Unlike the HPL phase in DSV thick films where the perforations are stacked in an AB sequence, ${ }^{25}$ the DSL-H in this work shows an AA stacking sequence according to the SEM and STEM images in Figure 1 and 4, respectively. The AA sequence refers to the perforations aligned above each other in adjacent lamellae, i.e. cylinders penetrating orthogonal to the lamellae. This is the same structure as we proposed in Ref (23) and it is also the structure observed in thick films of DSM blended with PDMSB- $b$-PS, which results in $f_{\mathrm{PDMSB}} / f_{\mathrm{PS}} / f_{\mathrm{PMMA}}=0.31 / 0.45 / 0.24 .{ }^{43}$ Thus, the two DSL-H here and the DSM blend in Ref (43) have similar volume fractions and are found to exhibit AA stacked $\mathrm{Lam}_{/ /}-\mathrm{Hex} \perp$ under solvent annealing in $\mathrm{CHCl}_{3}$, a morphology which has not been reported in other BCPs.

We note that the demonstrated use of block-selective infiltration in TBTPs achieves TEM contrast enhancement in a similar manner to traditional polymer staining methods based on $\mathrm{OsO}_{4}$ and $\mathrm{RuO}_{4}$ reagents, with the former known to oxidize polymers with olefinic functionalities and the latter to react primarily with aromatic and unsaturated polymers. ${ }^{51-53}$ However, these methods are either very slow (e.g., even up to several days), ${ }^{52}$ or difficult to perform due to the high toxicity of these metal oxides, and it may be hard to predict the domains to be stained due to various other chemical and structural characteristics known to affect the preferential reactivity of blocks. ${ }^{53}$ On the other hand, infiltration synthesis, relying on a relatively straightforward Lewis acid-base 
interaction between infiltrating precursors and polymers, allows us to predict the domain to be preferentially infiltrated and is quick and safe to implement using a commercial ALD system. 


\section{CONCLUSION}

In summary, a vapor-phase infiltration synthesis was demonstrated to selectively deposit inorganic material ( $\mathrm{ZnO}$ ) into an organic block (PLA) of a triblock terpolymer film. The infiltration extends over film thicknesses of at least $260 \mathrm{~nm}$ and enables resolution of microdomain morphologies and volume fractions of the blocks. The triblock terpolymer, DSL, shows a transition from a three-color lamellar structure to perforated lamellae plus perpendicular core-shell cylinders on decreasing the volume fraction of the PDMSB. Environmental oxidation of the PDMSB domains formed $\mathrm{SiO}_{\mathrm{x}}$, and infiltration of the PLA domains with $\mathrm{ZnO}$ results in hierarchical $\mathrm{ZnO} /$ polymer/SiO $\mathrm{x}$ nanostructures. Infiltration synthesis therefore provides access to multicomponent nanocomposites with both lateral and vertical periodicities as well as a process for investigating complex 3D BCP morphologies. 


\section{EXPERIMENTAL SECTION}

Materials and Preparation of DSL Films. Four linear PDMSB- $b$-PS- $b$-PLA (DSL) triblock terpolymers with different volume fractions of each block in Table $\mathbf{1}$ were synthesized via the combination of anionic polymerization by sequential addition of monomers and ring opening polymerization using a strong organic base as catalyst (also called pseudo anionic polymerization). A hydroxyl-terminated poly(1,1-dimethylsilacyclobutane- $b$-styrene) (PDMSB- $b$-PS-OH) was first prepared by the sequential anionic polymerization of DMSB and styrene monomers in various compositions using tetrahydrofuran/heptane (1:1) as a solvent at $-48{ }^{\circ} \mathrm{C}$ accompanied by the addition of ethylene oxide. This reaction was terminated using degassed methanol in order to achieve the desired end-functionalized symmetric and asymmetric PDMSB- $b$-PS BCPs. The ring opening polymerization of $\mathrm{D}$, L-lactide was then performed in toluene at $80^{\circ} \mathrm{C}$ using PDMSB- $b$ PS-OH as macroinitiator in the presence of catalyst triazabicyclodecene (TBD).$^{54}$

180-260 nm thick DSL films were obtained by spin-coating $4 \mathrm{wt} \%$ DSL solutions in a mixture of tetrahydrofuran and propylene glycol monomethyl ether acetate (THF/PGMEA $=2: 1$ ) onto silicon substrates with native oxide layer. The as-cast film thickness was determined by the spin speed of 3,000 rpm and measured using a spectral reflectometer (FilMetrics F20-UV). All the solvents used in this study were purchased from Sigma Aldrich.

Solvent Vapor Annealing of DSL Films. Solvent vapor annealing was carried out using a continuous flow system where separate streams of nitrogen are bubbled through liquid solvents and the flow of each stream is regulated using a mass flow controller (MKS Inc., M100B). ${ }^{55}$ Ascast DSL films on substrates were placed inside the glass annealing chamber (volume $\sim 80 \mathrm{~cm}^{3}$ ) tightly sealed by a quartz plate along with a perfluoroelastomer O-ring (Markez Inc., Z1210), and 
were annealed in a vapor flow of $\mathrm{CHCl}_{3}$ and $\mathrm{N}_{2}$ for $\sim 1 \mathrm{~h}$ at ambient temperature. Solvent vapors of $\mathrm{CHCl}_{3}: \mathrm{N}_{2}=10 \mathrm{sccm}: 1 \mathrm{sccm}$ were introduced to achieve an optimal swelling ratio of $\sim 2.1$ and 1.9 for DSL-L and DSL-H, respectively which produced well-ordered DSL morphologies. The inflow tube was connected to a mixer fed by two flow channels: pure $\mathrm{N}_{2}$ gas (Airgas, Inc., $99.9997 \%$ purity) for diluting the vapor pressure of $\mathrm{CHCl}_{3}$ and a solvent bubbler which contained $\mathrm{CHCl}_{3}$. During the deswelling, swollen films were slowly dried over $10 \mathrm{~min}$ by stopping the vapor flow of $\mathrm{CHCl}_{3}$ and $\mathrm{N}_{2}$ followed by gently detaching the quartz lid from the chamber at room temperature.

ZnO Infiltration Synthesis in DSL Films. The infiltration synthesis of ZnO was carried out in a commercial ALD system (Cambridge Nanotech, Savannah S100) at $85^{\circ} \mathrm{C}$ using DEZ and TMA as metal organic precursors along with water as an oxidant. The infiltration synthesis process consists of $1 \mathrm{AlO}_{\mathrm{x}}$-priming cycle followed by $6 \mathrm{ZnO}$ infiltration cycles. The priming step consists of: exposure to TMA for 100 s under a static vacuum ( 350 Torr); chamber purging using $\mathrm{N}_{2}$ (100 $\mathrm{sccm}$ ) for $100 \mathrm{~s}$; exposure to water vapor ( 10 Torr) for $100 \mathrm{~s}$; and chamber purging using $\mathrm{N}_{2}$ $(100 \mathrm{sccm})$ for $100 \mathrm{~s}$. The following $\mathrm{ZnO}$ infiltration cycle consists of exposure to DEZ for 6 min under a static vacuum ( 1.7 Torr); chamber purging using $\mathrm{N}_{2}(100 \mathrm{sccm})$ for $2 \mathrm{~min}$; exposure to water vapor ( 10 Torr) for $6 \mathrm{~min}$; and chamber purging using $\mathrm{N}_{2}(100 \mathrm{sccm})$ for $2 \mathrm{~min}$.

Characterization of DSL Films. As-annealed DSL films in Figure 1 were first etched by RIE (Plasma-Therm 790) to enhance the imaging contrast followed by Zeiss Merlin high-resolution SEM characterization at $3 \mathrm{kV}$ from multiple angles. For the SEM images in plain view, the PDMSB surface layer and microdomains of organic blocks (PS and PLA) were selectively removed by RIE treatment with $\mathrm{CF}_{4}(5 \mathrm{~s}, 15$ mTorr, $50 \mathrm{~W})$ and $\mathrm{O}_{2}(30 \mathrm{~s}, 6$ mTorr, $90 \mathrm{~W})$, respectively. Terraced regions of PDMSB were obtained by covering the half of the sample, 
etching the other half with $\mathrm{CF}_{4}(60 \mathrm{~s}, 15 \mathrm{mTorr}, 50 \mathrm{~W})$ and $\mathrm{O}_{2}(30 \mathrm{~s}, 6 \mathrm{mTorr}, 90 \mathrm{~W})$, and imaging the boundary between these two regions. This process yields oxidized PDMSB patterns that exhibit the morphology of the PDMSB microdomains. The cross-sections were prepared by cracking the sample in liquid nitrogen followed by RIE with $\mathrm{O}_{2}(5 \mathrm{~s}, 6 \mathrm{mTorr}, 90 \mathrm{~W})$ and imaged after tilting the sample $70^{\circ}$ to the SEM detector. The as-infiltrated organic-inorganic hybrids were characterized by TEM (JEOL 2100; $200 \mathrm{kV}$ ), and scanning TEM (FEI Talos F200X; $200 \mathrm{kV}$; equipped with the EDS elemental mapping capability). The cross-sectional TEM samples were prepared by the standard in situ lift-out procedure using Ga ion milling in a focused ion beam system (FEI Helios 600 Nanolab). 


\section{ACKNOWLEDGMENTS}

The authors acknowledge the support of the Semiconductor Research Corporation and Tokyo Electron. This research used resources of the Center for Functional Nanomaterials, which is a U.S. DOE Office of Science Facility, at Brookhaven National Laboratory under Contract No. DESC0012704.

\section{ASSOCIATED CONTENT}

\section{Supporting Information}

The Supporting Information is available free of charge on the ACS Publications website.

Additional information on SAXS patterns for the bulk morphologies, top view SEM images showing the vertical core-shell cylinders, and cross-sectional TEM/STEM images with EDS areal elemental mappings for DSL-L and DSL-H films

\section{AUTHOR INFORMATION}

\section{Corresponding Author}

*E-mail: caross@mit.edu (C.A.R.).

\section{Notes}

The authors declare no competing financial interest 


\section{REFERENCES}

1. Shulaker, M. M.; Hills, G.; Park, R. S.; Howe, R. T.; Saraswat, K.; Wong, H.-S. P.; Mitra, S. Three-Dimensional Integration of Nanotechnologies for Computing and Data Storage on a Single Chip. Nature 2017, 547, 74-78.

2. Javey, A.; Nam, S. W.; Friedman, R. S.; Yan, H.; Lieber, C. M. Layer-by-Layer Assembly of Nanowires for Three-Dimensional, Multifunctional Electronics. Nano Lett. 2007, 7, 773-777.

3. Li, H.; Li, X.; Guo, D.; Lou, L.; Li, W.; Zhang, X. Three-Dimensional Self-Assembly of Core/Shell-Like Nanostructures for High-Performance Nanocomposite Permanent Magnets. Nano Lett. 2016, 16, 5631-5638.

4. Fernández-Pacheco, A.; Streubel, R.; Fruchart, O.; Hertel, R.; Fischer, P.; Cowburn, R. P. ThreeDimensional Nanomagnetism. Nat. Commun. 2017, 8, 15756.

5. von Freymann, G.; Ledermann, A.; Thiel, M.; Staude, I.; Essig, S.; Busch, K.; Wegener, M. Three-Dimensional Nanostructures for Photonics. Adv. Funct. Mater. 2010, 20, 1038-1052.

6. Castles, F.; Day, F. V.; Morris, S. M.; Ko, D-H.; Gardiner, D. J.; Qasim, M. M.; Nosheen, S.; Hands, P. J. W.; Choi, S. S.; Friend, R. H.; Coles, H. J. Blue-Phase Templated Fabrication of Three-Dimensional Nanostructures for Photonic Applications. Nat. Mater. 2012, 11, 599-603.

7. Ross, C. A.; Berggren, K. K.; Cheng, J. Y.; Jung, Y. S.; Chang, J.-B. Three-Dimensional Nanofabrication by Block Copolymer Self-Assembly. Adv. Mater. 2014, 26, 4386-4396.

8. Tavakkoli K. G., A.; Gotrik, K. W.; Hannon, A. F.; Alexander-Katz, A.; Ross, C. A.; Berggren, K. K. Templating Three-Dimensional Self-Assembled Structures in Bilayer Block Copolymer Films. Science 2012, 336, 1294-1298.

9. Oh, J.; Suh, H. S.; Ko, Y.; Nah, Y.; Lee, J.-C.; Yeom, B.; Char, K.; Ross, C. A.; Son, J. G. Universal Perpendicular Orientation of Block Copolymer Microdomains Using a Filtered Plasma. Nat. Commun. 2019, 10, 2912.

10. Rahman, A.; Majewski, P. W.; Doerk, G.; Black, C. T.; Yager, K. G. Non-Native ThreeDimensional Block Copolymer Morphologies. Nat. Commun. 2016, 7, 13988.

11. Cheng, L.-C.; Simonaitis, J. W.; Gadelrab, K. R.; Tahir, M.; Ding, Y.; Alexander-Katz, A.; Ross, C. A. Imparting Superhydrophobicity with a Hierarchical Block Copolymer Coating. Small 2020, 16, 1905509.

12. Chuang, V. P.; Cheng, J. Y.; Savas, T. A.; Ross, C. A. Three-Dimensional Self-Assembly of Spherical Block Copolymer Domains into V-Shaped Grooves. Nano Lett. 2006, 6, 2332-2337. 
13. Lee, S.; Cheng, L.-C.; Gadelrab, K. R.; Ntetsikas, K.; Moschovas, D.; Yager, K. G.; Avgeropoulos, A.; Alexander-Katz, A.; Ross, C. A. Double-Layer Morphologies from a SiliconContaining ABA Triblock Copolymer. ACS Nano 2018, 12, 6193-6202.

14. Ren, Y.; Zou, Y.; Liu, Y.; Zhou, X.; Ma, J.; Zhao, D.; Wei, G.; Ai, Y.; Xi, S.; Deng, Y. Synthesis of Orthogonally Assembled 3D Cross-Stacked Metal Oxide Semiconducting Nanowires. Nat. Mater. 2020, 19, 203-211.

15. Choi, H. K.; Aimon, N. M.; Kim, D. H.; Sun, X. Y.; Gwyther, J.; Manners, I.; Ross, C. A. Hierarchical Templating of a $\mathrm{BiFeO}_{3}-\mathrm{CoFe}_{2} \mathrm{O}_{4}$ Multiferroic Nanocomposite by a Triblock Terpolymer Film. ACS Nano 2014, 8, 9248-9254.

16. Chuang, V. P.; Gwyther, J.; Mickiewicz, R. A.; Manners, I.; Ross, C. A. Templated SelfAssembly of Square Symmetry Arrays from an ABC Triblock Terpolymer. Nano Lett. 2009, 9 , 4364-4369.

17. Son, J. G.; Gwyther, J.; Chang, J.-B.; Berggren, K. K.; Manners, I.; Ross, C. A. Highly Ordered Square Arrays from a Templated ABC Triblock Terpolymer. Nano Lett. 2011, 11, 2849-2855.

18. Robbins, S. W.; Beaucage, P. A.; Sai1, H.; Tan, K. W.; Werner, J. G.; Sethna, J. P.; DiSalvo, F. J.; Gruner, S. M.; Van Dover, R. B.; Wiesner, U. Block Copolymer Self-Assembly-Directed Synthesis of Mesoporous Gyroidal Superconductors. Sci. Adv. 2016, 2, e1501119.

19. Docampo, P.; Stefik, M.; Guldin, S.; Gunning, R.; Yufa, N. A.; Cai, N.; Wang, P.; Steiner, U.; Wiesner, U.; Snaith, H. J. Triblock-Terpolymer-Directed Self-Assembly of Mesoporous $\mathrm{TiO}_{2}$ : High-Performance Photoanodes for Solid-State Dye-Sensitized Solar Cells. Adv. Energy Mater. 2012, 2, 676-682.

20. Vignolini, S.; Yufa, N. A.; Cunha, P. S.; Guldin, S.; Rushkin, I.; Stefik, M.; Hur, K.; Wiesner, U.; Baumberg, J. J.; Steiner, U. A 3D Optical Metamaterial Made by Self-Assembly. Adv. Mater. 2012, 24, OP23-OP27.

21. Löbling, T. I.; Borisov, O.; Haataja, J. S.; Ikkala, O.; Gröschel, A. H.; Müller, A. H. E. Rational Design of ABC Triblock Terpolymer Solution Nanostructures with Controlled Patch Morphology. Nat. Commun. 2016, 7, 12097.

22. Löbling, T. I.; Hiekkataipale, P.; Hanisch, A.; Bennet, F.; Schmalz, H.; Ikkala, O.; Groschel, A. H.; Müller, A. H.E. Bulk Morphologies of Polystyrene-block-Polybutadiene-block-Poly(tertbutyl methacrylate) Triblock Terpolymers. Polymer 2015, 72, 479-489.

23. Lee, S.; Cheng, L.-C.; Yager, K. G.; Mumtaz, M.; Aissou, K.; Ross, C. A. In Situ Study of ABC Triblock Terpolymer Self-Assembly under Solvent Vapor Annealing. Macromolecules 2019, 52, 1853-1863. 
24. Nunns, A.; Ross, C. A.; Manners, I. Synthesis and Bulk Self-Assembly of ABC Star Terpolymers with a Polyferrocenylsilane Metalloblock. Macromolecules 2013, 46, 2628-2635.

25. Aissou, K.; Mumtaz, M.; Bouzit, H.; Pecastaings, G.; Portale, G.; Fleury, G.; Hadziioannou, G. Bicontinuous Network Nanostructure with Tunable Thickness Formed on Asymmetric Triblock Terpolymer Thick Films. Macromolecules 2019, 52, 4413-4420.

26. Gu, X.; Gunkel, I.; Russell, T. P. Pattern Transfer Using Block Copolymers. Phil. Trans. $R$. Soc. A 2013, 371, 20120306.

27. Lee, S.-M.; Pippel, E.; Gösele, U.; Dresbach, C.; Qin, Y.; Chandran, C. V.; Bräuniger, T.; Hause, G.; Knez, M. Greatly Increased Toughness of Infiltrated Spider Silk. Science 2009, 324, 488-492.

28. Gong, B.; Peng, Q.; Jur, J. S.; Devine, C. K.; Lee, K.; Parsons, G. N. Sequential Vapor Infiltration of Metal Oxides into Sacrificial Polyester Fibers: Shape Replication and Controlled Porosity of Microporous/Mesoporous Oxide Monoliths. Chem. Mater. 2011, 23, 3476-3485.

29. Peng, Q.; Tseng, Y.-C.; Darling, S. B.; Elam, J. W. Nanoscopic Patterned Materials with Tunable Dimensions via Atomic Layer Deposition on Block Copolymers. Adv. Mater. 2010, 22, 5129-5133.

30. Peng, Q.; Tseng, Y.-C.; Darling, S. B.; Elam, J. W. A Route to Nanoscopic Materials via Sequential Infiltration Synthesis on Block Copolymer Templates. ACS Nano 2011, 5, 4600-4606.

31. Segal-Peretz, T.; Winterstein, J.; Doxastakis, M.; Ramirez-Hernandez, A.; Biswas, M.; Ren, J.; Suh, H. S.; Darling, S. B.; Liddle, J. A.; Elam, J. W.; de Pablo, J. J.; Zaluzec, N. J.; Nealey, P. F. Characterizing the Three-Dimensional Structure of Block Copolymers via Sequential Infiltration Synthesis and Scanning Transmission Electron Tomography. ACS Nano 2015, 9, 5333-5347.

32. Cummins, C.; Ghoshal, T.; Holmes, J. D.; Morris, M. A. Strategies for Inorganic Incorporation using Neat Block Copolymer Thin Films for Etch Mask Function and Nanotechnological Application. Adv. Mater. 2016, 28, 5586-5618.

33. Chai, J.; Buriak, J. M. Using Cylindrical Domains of Block Copolymers To Self-Assemble and Align Metallic Nanowires. ACS Nano 2008, 2, 489-501.

34. Subramanian, A.; Doerk, G.; Kisslinger, K.; Yi, D. H.; Grubbs, R. B.; Nam, C.-Y. ThreeDimensional Electroactive ZnO Nanomesh Directly Derived from Hierarchically Self-Assembled Block Copolymer Thin Films. Nanoscale 2019, 11, 9533-9546.

35. Subramanian, A.; Tiwale, N.; Nam, C.-Y. Review of Recent Advances in Applications of Vapor-Phase Material Infiltration Based on Atomic Layer Deposition. JOM 2019, 71, 185-196. 
36. Subramanian, A.; Tiwale, N.; Doerk, G.; Kisslinger, K.; Nam, C.-Y. Enhanced Hybridization and Nanopatterning via Heated Liquid-Phase Infiltration into Self-Assembled Block Copolymer Thin Films. ACS Appl. Mater. Interfaces 2020, 12, 1444-1453.

37. Aissou, K.; Mumtaz, M.; Marcasuzaa, P.; Brochon, C.; Cloutet, E.; Fleury, G.; Hadziioannou, G. Highly Ordered Nanoring Arrays Formed by Templated Si-Containing Triblock Terpolymer Thin Films, Small 2017, 13, 1603184.

38. Waldman, R. Z.; Mandia, D. J.; Yanguas-Gil, A.; Martinson, A. B. F.; Elam, J. W.; Darling, S. B. The Chemical Physics of Sequential Infiltration Synthesis-A Thermodynamic and Kinetic Perspective. J. Chem. Phys. 2019, 151, 190901.

39. Peng, Q.; Tseng, Y.-C.; Long, Y.; Mane, A. U.; DiDona, S.; Darling, S. B.; Elam, J. W. Effect of Nanostructured Domains in Self-Assembled Block Copolymer Films on Sequential Infiltration Synthesis. Langmuir 2017, 33, 13214-13223.

40. Kim, E.; Vaynzof, Y.; Sepe, A.; Guldin, S.; Scherer, M.; Cunha, P.; Roth, S. V.; Steiner, U. Gyroid-Structured 3D ZnO Networks Made by Atomic Layer Deposition. Adv. Funct. Mater. 2014, 24, 863-872.

41. Rahman, A.; Ashraf, A.; Xin, H.; Tong, X.; Sutter, P.; Eisaman, M. D.; Black, C. T. Sub-50nm Self-Assembled Nanotextures for Enhanced Broadband Antireflection in Silicon Solar Cells. Nat. Commun. 2015, 6, 5963.

42. Akyildiz, H. I.; Stano, K. L.; Roberts, A. T.; Everitt, H. O.; Jur, J. S. Photoluminescence Mechanism and Photocatalytic Activity of Organic-Inorganic Hybrid Materials Formed by Sequential Vapor Infiltration. Langmuir 2016, 32, 4289-4296.

43. Aissou, K.; Mumtaz, M.; Demazy, N.; Pécastaings, G.; Fleury, G.; Hadziioannou, G. Periodic Bicontinuous Structures Formed on the Top Surface of Asymmetric Triblock Terpolymer Thick Films. ACS Macro Lett. 2019, 8, 923-930.

44. Kamcev, J.; Germack, D. S.; Nykypanchuk, D.; Grubbs, R. B.; Nam, C.-Y.; Black, C. T. Chemically Enhancing Block Copolymers for Block-Selective Synthesis of Self-Assembled Metal Oxide Nanostructures. ACS Nano 2013, 7, 339-346.

45. Ocola, L. E.; Connolly, A.; Gosztola, D. J.; Schaller, R. D.; Yanguas-Gil, A. Infiltrated Zinc Oxide in Poly(methyl methacrylate): An Atomic Cycle Growth Study. J. Phys. Chem. C 2017, 121, 1893-1903.

46. Yu, Y.; Li, Z.; Wang, Y.; Gong, S.; Wang, X. Sequential Infiltration Synthesis of Doped Polymer Films with Tunable Electrical Properties for Efficient Triboelectric Nanogenerator Development. Adv. Mater. 2015, 27, 4938-4944. 
47. Gong, B.; Spagnola, J. C.; Parsons, G. N. Hydrophilic Mechanical Buffer Layers and Stable Hydrophilic Finishes on Polydimethylsiloxane Using Combined Sequential Vapor Infiltration and Atomic/Molecular Layer Deposition. J. Vac. Sci. Technol. A 2012, 30, 01A156.

48. Leng, C. Z.; Losego, M. D. Vapor Phase Infiltration (VPI) for Transforming Polymers into Organic-Inorganic Hybrid Materials: A Critical Review of Current Progress and Future Challenges. Mater. Horiz. 2017, 4, 747-771.

49. Waldman, R. Z.; Jeon, N.; Mandia, D. J.; Heinonen, O.; Darling, S. B.; Martinson, A. B. F. Sequential Infiltration Synthesis of Electronic Materials: Group 13 Oxides via Metal Alkyl Precursors. Chem. Mater. 2019, 31, 5274-5285.

50. She, Y.; Lee, J.; Lee, B.; Diroll, B.; Scharf, T.; Shevchenko, E. V.; Berman, D. Effect of the Micelle Opening in Self-assembled Amphiphilic Block Co-polymer Films on the Infiltration of Inorganic Precursors. Langmuir 2019, 35, 796-803.

51. Wang, Y.; Coombs, N.; Turak, A.; Lu, Z.-H.; Manners, I.; Winnik, M. A. Interfacial Staining of a Phase-Separated Block Copolymer with Ruthenium Tetroxide. Macromolecules 2007, 40, 1594-1597.

52. Loo, Y.-L.; Register, R. A.; Adamson, D. H. Direct Imaging of Polyethylene Crystallites within Block Copolymer Microdomains. J. Polym. Sci. B Polym. Phys. 2000, 38, 2564-2570.

53. Trent, J. S.; Scheinbeim, J. I.; Couchman, P. R. Ruthenium Tetraoxide Staining of Polymers for Electron Microscopy. Macromolecules 1983, 16, 589-598.

54. Simón, L.; Goodman, J. M. The Mechanism of TBD-Catalyzed Ring-Opening Polymerization of Cyclic Esters. J. Org. Chem. 2007, 72, 9656-9662.

55. Gotrik, K. W.; Hannon, A. F.; Son, J. G.; Keller, B.; Alexander-Katz, A.; Ross, C. A. Morphology Control in Block Copolymer Films Using Mixed Solvent Vapors. ACS Nano 2012, 6, 8052-8059. 
TABLE OF CONTENTS GRAPHIC

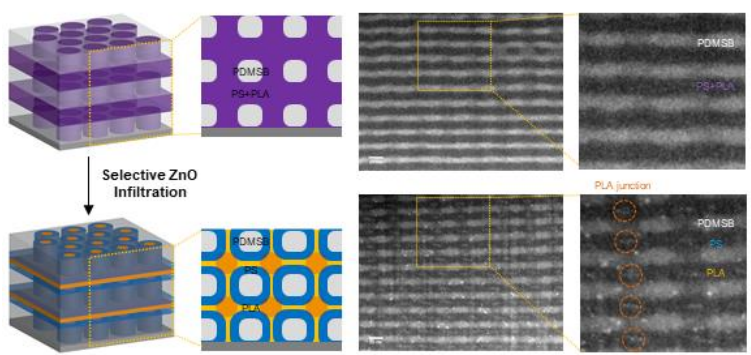

